\title{
Sistemas de localização indoor utilizando Bluetooth Low Energy: Uma revisão sistemática
}

\author{
Matheus da Silva Rodrigues ${ }^{1}$, Sérgio Teixeira de Carvalho ${ }^{1}$ \\ ${ }^{1}$ Instituto de Informática - Universidade Federal de Goiás (UFG) \\ \{matheusrodrigues, sergio\} @inf.ufg.br
}

\begin{abstract}
Indoor location is essential because there is a growth of applications that make use of this. Consequently, the need to locate users efficiently in these environments also arises. Therefore, this paper performs a systematic review of existing methods of indoor location. With the definition and application of a research method, the review intends to determine how the current state of the art of this subject is.
\end{abstract}

Resumo. A localização indoor mostra-se essencial visto que há um crescimento de aplicações que fazem o uso desta. Consequentemente, a necessidade de localizar usuários de forma eficiente nestes ambientes também surge. Por isto, este artigo realiza uma revisão sistemática dos métodos existentes de localização indoor. Com a definição e a aplicação de um método de pesquisa, a revisão tem o intuito de determinar como se encontra o atual estado da arte deste assunto.

\section{Introdução}

A criação e aplicação do GPS, bem como a sua integração com os smartphones, possibilitaram considerável avanço no que diz respeito à localização, tornando-o indispensável às aplicações que necessitem estabelecer a posição do usuário. No entanto, o GPS atinge uma precisão aceitável somente em ambientes externos (outdoor), não sendo adequado para ambientes internos (indoor), em razão da atenuação e reflexão dos sinais [Li et al. 2016].

Nessas circunstâncias, a tecnologia Bluetooth Low Energy (BLE) apresenta-se com um forte potencial para lidar com este problema, pois oferece um baixo preço e uma alta usabilidade [Cay et al. 2017]. Tão importante quanto a ferramenta, o conhecimento e o emprego de métodos, técnicas e algoritmos fazem-se necessários para que a localização indoor possa ser estabelecida.

O objetivo desse artigo é apresentar os resultados de uma revisão sistemática conduzida para identificar o estado da arte dos métodos e algoritmos que têm sido empregados em aplicações de localização indoor de pessoas e objetos com o uso da tecnologia BLE. O artigo está estruturado em outras 4 seções. A Seção 2 apresenta a contextualização e o funcionamento dos beacons BLE. A Seção 3 descreve o processo de revisão sistemática realizado. A Seção 4, por sua vez, apresenta os resultados e uma discussão relacionada a um problema da localização indoor. Por fim, a Seção 5 traz a conclusão.

\section{Fundamentação teórica}

Devido ao baixo custo e alta usabilidade do BLE [Li et al. 2016], o seu potencial como uma ferramenta eficaz para localização indoor tem despertado o interesse do meio acadêmico-científico [Chandel et al.2016]. Um beacon BLE, consiste em um chipset, uma bateria e uma antena. Trata-se de um dispositivo de transmissão sem qualquer necessidade de conexão à internet, que emite com periodicidade pacotes Bluetooth com informações que podem ser usadas pelos receptores em intervalos regulares de tempo [Cay et al. 2017].

Utilizando o Received Signal Strength Indicator (RSSI) como parâmetro de referência para medir distâncias entre o emissor e o receptor, é possível determinar a localização do usuário utilizando técnicas já conhecidas e usadas por sistemas de localização baseadas em Wi-Fi [Chandel et al.2016]. Todavia, o RSSI não é um parâmetro estável, sendo altamente sensível a pequenas mudanças no ambiente a ser analisado [Filípek and Kovarova 2016]. Devido a alta instabilidade dos sinais fornecidos pelos beacons, uma etapa de pré-processamento destes dados pode ser feita a fim de linearizar o modelo matemático referente ao RSSI. Assim que os valores RSSI são obtidos e processados, o algoritmo de localização entra em ação. De uma forma geral, estes algoritmos 
realizam os seguintes passos [Davidson and Piché 2017]: i) Captura dos valores RSSI; ii) Préprocessamento dos dados; iii) Cálculo da distância; iv) Cálculo da posição atual.

\section{Materiais e métodos}

Essa seção apresenta a metodologia de pesquisa definida e aplicada para a realização desta revisão, com destaque para a definição do protocolo, a seleção dos artigos e a análise qualitativa.

$\mathrm{O}$ protocolo de pesquisa foi definido com base no modelo proposto por [Budgen and Brereton 2006], o qual foi aplicado nas bases IEEE, ACM e ScienceDirect. Duas questões de pesquisa foram elaboradas, e, a partir delas, a string de busca e os critérios de elegibilidade foram definidos. QP1) Quais métodos são utilizados para a realização da localização; QP2) Qual a precisão atingida por estes métodos.

A seguinte string de busca foi definida para a coleta dos artigos nas referidas bases de dados:

Como resultado, 395 artigos foram encontrados, dos quais 85 foram selecionados a partir da

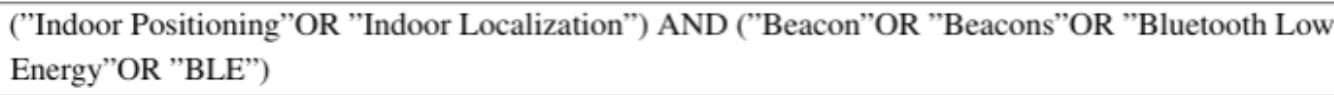

aplicação dos seguintes critérios de elegibilidade: i) Artigo científico; ii) Artigo escrito em português ou inglês; iii) Artigo apresenta uma aplicação ou sistema; iv) Artigo faz uso de BLE; v) Artigo trata de localização indoor. Estes critérios foram aplicados na leitura dos títulos, abstracts e keywords dos artigos.

A análise qualitativa foi, então, realizada nos 85 artigos selecionados. O intuito é delimitar o escopo dos trabalhos que possuem notória relevância para as questões de pesquisa. Isto foi alcançado definindo fatores essenciais para a revisão e verificando se os artigos os possuíam ou não. A Figura 1 apresenta como isto foi aplicado.

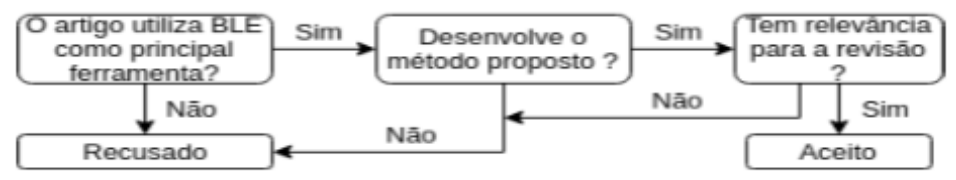

\section{Figura 1. Fluxograma de seleção na análise qualitativa}

Ao fim deste processo, 22 artigos foram aceitos para a revisão final. Estes tiveram seus conteúdos lidos por completo a fim de responder às questões de pesquisa.

\section{Resultados e discussão}

Em relação a QP1, os métodos utilizados pelos autores foram divididos em 5 categorias, como apresentadas na Tabela 1: Trilateração, Triangulação, Proximidade, Probabilístico e Reconhecimento de Padrões.

\begin{tabular}{|c|c|c|c|c|}
\hline Trilateração & Triangulação & Proximidade & Probabilístico & Reconhecimento de Padrões \\
\hline $\begin{array}{c}\text { [Cay et al. 2017] } \\
\text { [Noertjahyana et al. 2017] } \\
\text { [Kuxdorf-Alkirata and Brückmann 2016] } \\
\text { [Ndzukula et al. 2017] } \\
\text { [Chandel et al. 2016] } \\
\text { [Thaljaoui et al. 2015] }\end{array}$ & $\begin{array}{c}\text { [Kanzaki and Fujita 2016] } \\
\text { [Li et al. 2016] }\end{array}$ & $\begin{array}{l}\text { [Memon et al. 2017] } \\
\text { [Kyritsis et al. 2016] }\end{array}$ & $\begin{array}{l}\text { [Filípek and Kovarova 2016] } \\
\text { [Hou and Arslan 2017] }\end{array}$ & $\begin{array}{c}\text { [Luca and Alberto 2016] } \\
\text { [Pu and You 2018] } \\
\text { [Benaissa et al. 2018] } \\
\text { [Tabbakha et al. 2017] } \\
\text { [Xiao et al. 2017] } \\
\text { [Lu et al. 2017] } \\
\text { [Jondhale and Deshpande 2019] } \\
\text { [Peng et al. 2016] } \\
\text { [Palumbo et al. 2015] } \\
\text { [Ma et al. 2017] }\end{array}$ \\
\hline
\end{tabular}

Tabela 1. Distribuição dos métodos utilizados pelos autores

Quanto à QP2, a Tabela 2 apresenta a distribuição dos artigos considerando a perspectiva da precisão alcançada nas variadas soluções descritas pelos autores. Pelo que foi observado, pode-se tratar a precisão em termos de cômodo (onde somente o ambiente/cômodo em que o usuário se encontra é identificado), em termos de metros (onde o erro da precisão está na casa dos metros) e em termos de centímetros. 
Tabela 2. Distribuição dos métodos utilizados pelos autores

\begin{tabular}{|c|c|c|}
\hline Cômodo & Metros & Centímetros \\
\hline & [Noertjahyana et al. 2017], [Peng et al. 2016], & [Hou and Arslan 2017], \\
& [Li et al. 2016], [Lu et al. 2017], & [Jondhale and Deshpande 2019], [Chandel et al. 2016], \\
[Kyritsis et al. 2016], & [Ndzukula et al. 2017],[Cay et al. 2017], \\
[Memon et al. 2017] & [Tabbakha et al. 2017], [Kanzaki and Fujita 2016], \\
& [Luca and Alberto 2016], [Pu and You 2018], \\
& [Xiao et al. 2017] e [Palumbo et al. 2015] & [Benaissa et al. 2018], \\
& [Filípek and Kovarova 2016], \\
& [Thaljaoui et al. 2015] e [Ma et al. 2017] \\
\hline
\end{tabular}

Durante a condução da revisão, foi observado que todos os métodos sofrem de um problema relacionado a precisão. O problema ocorre pela forma como os métodos funcionam, pois necessitam de valores de referência RSSI dos beacons em uma determinada posição ou distância. Quando há uma alteração no ambiente, como, por exemplo, a movimentação de pessoas ou objetos, os valores de referência RSSI tendem a mudar, causando um erro na precisão dos métodos. Tomando como base os valores RSSI, qualquer método que os utilize como referência está suscetível à baixa precisão em ambientes dinâmicos.

\section{Conclusão}

Este artigo realizou uma revisão sistemática com o objetivo de perceber o atual estado da arte da localização indoor utilizando beacons BLE. Uma metodologia foi defina e aplicada em bases de dados previamente escolhidas, obtendo os artigos finais para a leitura completa e extração de dados. Foi notado que os métodos mais utilizados são os de trilateração ou reconhecimento de padrões, ambos atingindo em sua maioria a precisão em termos de metros. Um dos maiores desafios observados quanto à localização indoor é o desenvolvimento de um método que alcance alta precisão em ambientes dinâmicos.

\section{Referências}

Benaissa, B., Hendrichovsky, F., Yishida, K., Koppen, M., and Sincak, P. (2018). Phone application for indoor localization based on ble signal fingerprint. In 2018 9th IFIP Int. Conf. on New Techs., Mobility and Security (NTMS), pages 1-5.

Budgen, D. and Brereton, P. (2006). Performing systematic literature reviews in software engineering. In Proceedings of the 28th Int. Conf. on Software Engineering, ICSE '06, pages 1051-1052, New York, NY, USA. ACM.

Cay, E., Mert, Y., Bahcetepe, A., Akyazi, B. K., and Ogrenci, A. S. (2017). Beacons for indoor positioning. In 2017 Int. Conf. on Engineering and Technology (ICET), pages 1-5.

Chandel, V., Ahmed, N., Arora, S., and Ghose, A. (2016). Inloc: An end-to-end robust indoor localization and routing solution using mobile phones and ble beacons. In 2016 Int. Conf. on Indoor Positioning and Indoor Navigation (IPIN), pages 1-8.

Davidson, P. and Piche, R. (2017). A survey of selected indoor positioning methods for smartphones. IEEE Communications Surveys Tutorials, 19(2):1347-1370.

Filípek, P. and Kovarova, A. (2016). Indoor localization based on beacons and calculated by particle filter. In Proceedings of the 17th Int. Conf. on Computer Systems and Techs. 2016, CompSysTech '16, pages 269-276, New York, NY, USA. ACM.

Hou, X. and Arslan, T. (2017). Monte carlo localization algorithm for indoor positioning using bluetooth low energy devices. In 2017 Int. Conf. on Localization and GNSS (ICL-GNSS), pages 1-6.

Jondhale, S. R. and Deshpande, R. S. (2019). Grnn and kf framework based real time target tracking using psoc ble and smartphone. Ad Hoc Networks, 84:19 - 28.

Kanzaki, R. and Fujita, S. (2016). Indoor positioning based on the ibeacon framework with gaussian weight functions.

In 2016 IEEE Intl Conference on Computational Science and Engineering (CSE) and IEEE Intl Conference on

Embedded and Ubiquitous Computing (EUC) and 15th Intl Symposium on Distributed Computing and Applications for Business Engineering (DCABES), pages 158-165.

Kuxdorf-Alkirata, N. and Bruckmann, D. (2016). Reliable and low-cost indoor localization based on bluetooth low energy. In 2016 3rd Int. Symposium on Wireless Systems within the Conf. on Intelligent Data Acquisition and Advanced Comp. Systems, pages 92-96.

Kyritsis, A. I., Kostopoulos, P., Deriaz, M., and Konstantas, D. (2016). A ble-based probabilistic room- 
level localization method. In 2016 Int. Conf. on Localization and GNSS, pages 1-6.

Li, X., Xu, D., Wang, X., and Muhammad, R. (2016). Design and implementation of indoor positioning system based on ibeacon. In 2016 International Conference on Audio, Language and Image Processing (ICALIP), pages 126-130.

Lu, T. T., Liu, Y. J., Ciou, J. J., and Lu, C. H. (2017). Feature selection difference matching method for indoor positioning. In Proceedings of the Second International Conference on Internet of Things, Data and Cloud Computing, ICC '17, pages 116:1-116:5, New York, NY, USA. ACM.

Luca, D. G. and Alberto, M. (2016). From proximity to accurate indoor localization for context awareness in mobile museum guides. In Proceedings of the 18th Int. Conf. on Human-Computer Interaction with Mobile Devices and Services Adjunct, MobileHCI '16, pages 1002-1009, New York, NY, USA. ACM.

Ma, Z., Poslad, S., Bigham, J., Zhang, X., and Men, L. (2017). A ble rssi ranking based indoor positioning system for generic smartphones. In 2017 Wireless Tele. Symposium (WTS), pages 1-8.

Memon, S., Memon, M. M., Shaikh, F. K., and Laghari, S. (2017). Smart indoor positioning using ble technology. In 2017 4th IEEE Int. Conf. on Engineering Techs. and Applied Sciences (ICETAS), pages $1-5$.

Ndzukula, S. G., Ramotsoela, T. D., Silva, B. J., and Hancke, G. P. (2017). A bluetooth low energy based system for personnel tracking. In IECON 2017 - 43rd Annual Conference of the IEEE Industrial Electronics Society, pages 8435-8440.

Noertjahyana, A., Wijayanto, I. A., and Andjarwirawan, J. (2017). Development of mobile indoor positioning system application using android and bluetooth low energy with trilateration method. In 2017 Int. Conf. on Soft Comp., Intelligent System and Information Tech. (ICSIIT), pages 185-189.

Palumbo, F., Barsocchi, P., Chessa, S., and Augusto, J. C. (2015). A stigmergic approach to indoor localization using bluetooth low energy beacons. In 2015 12th IEEE Int. Conf on Advanced Video and Signal Based Surveillance (AVSS), pages 1-6.

Peng, Y., Fan, W., Dong, X., and Zhang, X. (2016). An iterative weighted knn (iw-knn) based indoor localization method in bluetooth low energy (ble) environment. In 2016 Int. IEEE Confs. on Ubiquitous Intelligence Comp., Advanced and Trusted Comp., Scalable Comp. and Communications, Cloud and Big Data Comp., Internet of People, and Smart World Congress, pages 794-800.

Pu, Y.-C. and You, P.-C. (2018). Indoor positioning system based on ble location fingerprinting with classification approach. Applied Mathematical Modelling, 62:654 - 663.

Tabbakha, N. E., Tan, W., and Ooi, C. (2017). Indoor location and motion tracking system for elderly assisted living home. In 2017 Int. Conf. on Robotics, Automation and Sciences (ICORAS), pages 1-4.

Thaljaoui, A., Val, T., Nasri, N., and Brulin, D. (2015). Ble localization using rssi measurements and iringla. In 2015 IEEE Int. Conf. on Industrial Technology (ICIT), pages 2178-2183.

Xiao, C., Yang, D., Chen, Z., and Tan, G. (2017). 3-d ble indoor localization based on denoising autoencoder. IEEE Access, 5:12751-12760. 